\title{
Warm asymmetric matter in the quark-meson coupling model
}

\author{
P. K. Panda, ${ }^{1,2}$ G. Krein, ${ }^{1}$ D. P. Menezes, ${ }^{2}$ and C. Providência ${ }^{3}$ \\ ${ }^{1}$ Instituto de Física Teórica, Universidade Estadual Paulista, Rua Pamplona 145, 01405-900 São Paulo, SP, Brazil \\ ${ }^{2}$ Departmento de Física, Universidade Federal de Santa Catarina, 88040-900 Florianópolis, SC, Brazil \\ ${ }^{3}$ Centro de Física Teórica, Departmento de Física, Universidade de Coimbra, Coimbra 3000, Portugal
}

(Received 1 April 2003; published 22 July 2003)

\begin{abstract}
In this work we study the warm equation of state of asymmetric nuclear matter in the quark-meson coupling model which incorporates explicitly quark degrees of freedom, with quarks coupled to scalar, vector, and isovector mesons. Mechanical and chemical instabilities are discussed as a function of density and isospin asymmetry. The binodal section, essential in the study of the liquid-gas phase transition is also constructed and discussed. The main results for the equation of state are compared with two common parametrizations used in the nonlinear Walecka model and the differences are outlined.
\end{abstract}

DOI: 10.1103/PhysRevC.68.015201

PACS number(s): 21.65.+f, 24.85.+p, 12.38.Lg

\section{INTRODUCTION}

The understanding of the properties of hot dense nuclear matter is an important problem in theoretical physics in the context of neutron stars [1] as well as in high-energy heavyion collisions experiments where nuclei undergo violent collisions [2]. Such collisions may produce states of nuclear matter at conditions far from those normally encountered in low energy collisions. For example, at high density or high temperature, nucleons in the nucleus may dissolve into quarks and gluons. At low temperature, which can be attained in medium-energy heavy-ion reactions, no such exotic state can be produced, but there is the possibility of interesting liquid-gas phase transitions leading to the breakup of heated nuclei into small clusters or droplets of nucleons [3]. Such phase transitions have been identified in multifragmentation experiments and in the crusts of neutron stars $[4,5]$. The possibilities of such phase transitions were previously considered by several authors using different approaches [6-8].

In the present paper, we employ the quark-meson coupling model (QMC) $[9,10]$ to investigate the liquid-gas phase transition in nuclear matter at different isospin asymmetries. In the QMC model, nuclear matter is described as a system of nonoverlapping MIT bags which interact through the effective scalar and vector mean fields, very much in the same way as in the Walecka model, or more generally quantum hadrodynamics (QHD) [11]. Many applications and extensions of the model have been made in the past years [12-15], including droplet formation at zero temperature [16]. A special improvement is related with a density dependent bag parameter. This model is known as modified QMC model (MQMC) $[17,18]$ and some of its characteristics are discussed in the last section of the present paper.

The crucial difference is that in the QMC, the effective meson fields couple directly to the confined quarks inside the nucleon bags instead to point like nucleons as in QHD. While the QMC model shares many similarities with QHDtype models, it, however, offers new opportunities for studying nuclear matter properties. One of the most attractive aspects of the model is that different phases of hadronic matter, from very low to very high baryon densities and tempera- tures, can be described within the same underlying model. In the QMC, matter at low densities and temperatures is a system of nucleons interacting through meson fields, with quarks and gluons confined within MIT bags. For matter at very high density and/or temperature, one expects that baryons and mesons dissolve and the entire system of quarks and gluons becomes confined within a single, big MIT bag. Another important aspect of the QMC is that the internal structure of the nucleon is introduced explicitly. Although the internal structure of nucleons can be effectively taken into account with an effective field theory employing hadronic degrees of freedom in a derivative expansion [19], it is clear that such an approach will fail to describe the transition to a quark-gluon phase. It is found that the equation of state (EOS) for infinite nuclear matter at zero temperature derived from the QMC model is much softer than the one obtained in the Walecka model [11]. Also, the QMC nucleon effective mass lies in the range $0.7-0.8$ of the free nucleon mass, which agrees with results derived from nonrelativistic analysis of scattering of neutrons from lead nuclei [20] and is larger than the effective nucleon mass in the Walecka model. At finite temperature, there arises yet another difference between predictions of QMC and QHD, namely, the behavior of the effective nucleon mass with the temperature at fixed baryon density. While in QHD-type models the nucleon mass always decreases with temperature, in the QMC it increases. The difference arises because of the explicit treatment of the internal structure of the nucleon in the QMC. When the bag is heated up, quark-antiquark pairs are excited in the interior of the bag, increasing the internal energy of the bag.

Here, we analyze the QMC model at finite temperature for different proton fractions. The proper treatment of the problem with isospin asymmetry requires not only isoscalar, scalar, and vector mesons fields, but also isovector meson fields. In particular, we investigate the consequences of the different thermal behavior of the nucleon mass in the QMC model on the liquid-gas phase transition. The results are also compared with the ones obtained with two commonly used parametrizations for a nonlinear Walecka model (NLWM), namely, NL3 [21] and TM1 [22]. We organize the present paper as follows. In Sec. II we describe the QMC model at finite temperature for asymmetric nuclear matter and discuss the 
binodal section. In Sec. III we present our numerical results and discuss differences with other approaches. Our summary is presented in Sec. IV.

\section{QMC AT FINITE TEMPERATURES}

\section{A. Asymmetric matter at finite temperature}

Recently, the QMC model has been generalized to finite temperatures taking a medium dependent bag constant [14]. In this generalized model, symmetric nuclear matter was considered, and hence only the interaction of quarks through the exchange of effective isoscalar scalar $(\sigma)$ and vector $(\omega)$ mesonic fields were taken into account. We now extend this model to asymmetric nuclear matter at finite temperatures and include the contribution of the isovector vector meson $\rho$, in addition to $\sigma$ and $\omega$.

In the QMC model, the nucleon in nuclear matter is assumed to be described by a static MIT bag in which quarks couple to effective meson fields, which are treated as classical in a mean field approximation. The quark field $\psi_{q}(\mathbf{r}, t)$ inside the bag then satisfies the Dirac equation

$$
\left[i \vec{\gamma} \cdot \vec{\partial}-\left(m_{q}^{0}-V_{\sigma}\right)-\gamma^{0}\left(V_{\omega}+\frac{1}{2} \tau_{3 q} V_{\rho}\right)\right] \psi_{q}(\mathbf{r}, t)=0,
$$

where $V_{\sigma}=g_{\sigma}^{q} \sigma_{0}, V_{\omega}=g_{\omega}^{q} \omega_{0}$, and $V_{\rho}=g_{\rho}^{q} b_{03}$; with $\sigma_{0}, \omega_{0}$, and $b_{03}$ being the classical meson fields. $g_{\sigma}^{q}, g_{\omega}^{q}$, and $g_{\rho}^{q}$ are the quark couplings with the $\sigma, \omega$, and $\rho$ mesons, respectively. $m_{q}^{0}$ is the current quark mass and $\tau_{3 q}$ is the third component of the Pauli matrices. In the present paper we consider nonstrange $q=u$ and $d$ quarks only.

At zero temperature, the normalized ground state ( $s$-state) wave function for a quark in the bag is given as

$$
\psi_{q}(\vec{r}, t)=N \exp \left(-i \frac{\epsilon_{q} t}{R}\right)\left(\begin{array}{c}
j_{0}(x r / R) \\
i \beta_{q} \vec{\sigma} \cdot \hat{r} j_{1}(x r / R)
\end{array}\right) \frac{\chi_{q}}{\sqrt{4 \pi}},
$$

where

$$
\beta_{q}=\sqrt{\frac{\Omega_{q}-R m_{q}^{*}}{\Omega_{q}+R m_{q}^{*}}}
$$

with

$$
\Omega_{q}=\sqrt{x^{2}+\left(R m_{q}^{*}\right)^{2}}, \quad m_{q}^{*}=m_{q}^{0}-g_{\sigma}^{q} \sigma,
$$

and $\chi_{q}$ is a Pauli spinor.

At finite temperatures, the three quarks inside the bag can be thermally excited to higher states, and also quarkantiquark pairs can be created. For simplicity, we assume that the bag describing the nucleon continues to remain in a spherical shape with radius $R$, which is now temperature dependent. The single-particle energies in units of $R^{-1}$ are given as

$$
\epsilon_{q}^{n \kappa}=\Omega_{q}^{n \kappa}+R\left(V_{\omega} \pm \frac{1}{2} V_{\rho}\right)
$$

for the quarks and

$$
\epsilon_{\bar{q}}^{n \kappa}=\Omega_{q}^{n \kappa}-R\left(V_{\omega} \pm \frac{1}{2} V_{\rho}\right)
$$

for the antiquarks, where the + sign is for $u$ quarks and for $d$ quarks, and

$$
\Omega_{q}^{n \kappa}=\sqrt{x_{n \kappa}^{2}+R^{2} m_{q}^{* 2}} .
$$

The eigenvalues $x_{n \kappa}$ for the state characterized by $n$ and $\kappa$ are determined by the boundary condition at the bag surface,

$$
i \gamma \cdot n \psi_{q}^{n \kappa}=\psi_{q}^{n \kappa} .
$$

Thus, the quark eigenvalues $x_{n \kappa}$ become modified by the surrounding nucleon medium at finite temperature.

The total energy from the quarks and antiquarks at finite temperature is

$$
E_{\mathrm{tot}}=\sum_{q, n, \kappa} \frac{\Omega_{q}^{n \kappa}}{R}\left(f_{n \kappa}^{q}+f_{n \kappa}^{\bar{q}}\right),
$$

where

$$
f_{n \kappa}^{q}=\frac{1}{e^{\left(\Omega_{q}^{\left.n \kappa / R-v_{q}\right) / T}+1\right.}}
$$

and

$$
f_{n \kappa}^{\bar{q}}=\frac{1}{e^{\left(\Omega_{q}^{n \kappa} / R+\nu_{q}\right) / T}+1},
$$

with $\nu_{q}$ being the effective quark chemical potential, related to the true quark chemical potential $\mu_{q}$ as

$$
\nu_{q}=\mu_{q}-V_{\omega}-m_{\tau}^{q} V_{\rho} .
$$

The bag energy now becomes

$$
E_{\mathrm{bag}}=E_{\mathrm{tot}}-\frac{Z}{R}+\frac{4 \pi}{3} R^{3} B
$$

where $B$ is the bag constant and $Z$ parametrizes the sum of the center-of-mass motion and gluonic corrections. Note that this center-of-mass correction is different from that of Ref. [17]. The effective nucleon mass is obtained from the bag energy and reads

$$
M_{N}^{*}=E_{\text {bag }} .
$$

The bag radius $R$ is determined by the equilibrium condition for the nucleon bag in the medium,

$$
\frac{\partial M_{N}^{*}}{\partial R}=0 .
$$

Once the bag radius is obtained, the effective nucleon mass is immediately determined. For a given temperature $T$ and scalar field $\sigma$, the effective quark chemical potentials $\nu_{q}, q$ $=u, d$ are determined from the total number of quarks of each type in the proton and in the neutron, i.e., 


$$
\begin{gathered}
n_{0}^{j}=\sum_{q, n, \kappa}\left(f_{n q}^{q}-f_{n q}^{\bar{q}}\right) \equiv 3, \\
n_{3}^{j}=\sum_{q, n, \kappa} 2 m_{\tau(q)}\left(f_{n q}^{q}-f_{n q}^{\bar{q}}\right) \equiv 2 m_{\tau(j)},
\end{gathered}
$$

for $j=p, n$.

From the last two expressions one can see that the masses for protons and neutrons are different, since the effective chemical potentials $\nu_{q}, q=u, d$ are not the same. Nevertheless, it is a reasonable approximation (for the purposes of the present paper) to take for the nucleon mass the average value, so that we are left only with Eq. (16), and hence $\nu_{u}$ $=\nu_{d}$. As a starting point, this approximation would go along the Walecka model which, with the most common parametrizations, does not distinguish the masses of protons and neutrons. The situation would be different for problems where the neutron-proton mass difference is the main issue, such as those discussed in Ref. [23]. We stress that for a fixed $T$ and $\sigma$ the quark distribution functions only depend on the effective chemical potentials $\nu_{q}$, which are determined by the constraints in Eqs. (16) and (17). Therefore, just as it happens at zero temperature, also at finite temperature the effective mass $M_{N}^{*}$ does not depend on $\omega$ and $b_{03}$.

Before we proceed, we note that we are not considering density/temperature dependence in the zero point energy parametrized by $Z$ because this would introduce extra, presently unknown, parameters. Blunden and Miller in [13] have proposed changing $Z$ through a linear dependence with the scalar field and concluded that for reasonable parameter ranges this has a little effect.

The total energy density at finite temperature $T$ and at finite baryon density $\rho_{B}$ is

$$
\begin{aligned}
\mathcal{E}= & \frac{2}{(2 \pi)^{3}} \sum_{i=p, n} \int d^{3} k\left[\epsilon^{*}\left(f_{i}+\bar{f}_{i}\right)+\mathcal{V}_{0 i}\left(f_{i}-\bar{f}_{i}\right)\right] \\
& +\frac{1}{2} m_{\sigma}^{2} \sigma^{2}-\frac{1}{2} m_{\omega}^{2} \omega^{2}-\frac{1}{2} m_{\rho}^{2} b_{03}^{2},
\end{aligned}
$$

where $f_{i}$ and $\bar{f}_{i}$ are the thermal distribution functions for the baryons and antibaryons,

$$
f_{i}=\frac{1}{e^{\left(\epsilon^{*}-\nu_{i}\right) / T}+1} \quad \text { and } \quad \bar{f}_{i}=\frac{1}{e^{\left(\epsilon^{*}+\nu_{i}\right) / T}+1}
$$

with $i=p, n$ and $\epsilon^{*}=\left(\vec{k}^{2}+M_{N}^{* 2}\right)^{1 / 2}$ the effective nucleon energy, $\nu_{i}=\mu_{i}-\mathcal{V}_{0 i}$ the effective baryon chemical potential, and $\mathcal{V}_{0 i}=g_{\omega} \omega+m_{\tau} g_{\rho} b_{03}\left(m_{\tau}= \pm 1 / 2\right.$, respectively, for protons and neutrons).

The thermodynamic grand potential density is defined as

$$
\Omega=\mathcal{E}-T \mathcal{S}-\sum_{i=p, n} \mu_{i} \rho_{i}
$$

with the entropy density $\mathcal{S}=S / V$ given by

$$
\begin{aligned}
\mathcal{S}= & -\sum_{i=p, n} \frac{2}{(2 \pi)^{3}} \int d^{3} k\left[f_{i} \ln f_{i}+\left(1-f_{i}\right) \ln \left(1-f_{i}\right)+\bar{f}_{i} \ln \bar{f}_{i}\right. \\
& \left.+\left(1-\bar{f}_{i}\right) \ln \left(1-\bar{f}_{i}\right)\right] .
\end{aligned}
$$

The proton or neutron density is given by

$$
\rho_{i}=\frac{2}{(2 \pi)^{3}} \int d^{3} k \quad\left(f_{i}-\bar{f}_{i}\right),
$$

so that the baryon density is $\rho=\rho_{p}+\rho_{n}$ and the (third component of) isospin density $\rho_{3}=\rho_{p}-\rho_{n}$. The proton fraction is defined as

$$
y_{p}=\frac{\rho_{p}}{\rho} \text {. }
$$

The pressure is the negative of $\Omega$, which after integration by parts can be written as

$$
\begin{aligned}
P= & \frac{1}{3} \sum_{i=p, n} \frac{2}{(2 \pi)^{3}} \int d^{3} k \frac{\mathbf{k}^{2}}{\epsilon^{*}(k)}\left(f_{i}+\bar{f}_{i}\right)-\frac{1}{2} m_{\sigma}^{2} \sigma^{2} \\
& +\frac{1}{2} m_{\omega}^{2} \omega^{2}+\frac{1}{2} m_{\rho}^{2} b_{03}^{2} .
\end{aligned}
$$

From the above expression the pressure depends explicitly on the meson mean fields $\sigma, \omega$, and $b_{03}$. It also depends on the nucleon effective mass $M_{N}^{*}$; which in turn also depends on the $\sigma$ field [see Eqs. (9)-(14)].

At a given temperature and for given $\rho_{p}, \rho_{n}$, the effective nucleon mass is known for given values of the meson fields, once the bag radius $R$ and the effective quark chemical potentials $\nu_{q}$ are calculated by using Eqs. (15) and (16), respectively. Maximizing the pressure with respect to the fields for a given temperature $T$ and given chemical potentials $\mu_{i}$, we obtain the following equations for the $\omega$ and $b_{03}$ fields:

$$
\omega=\frac{g_{\omega}}{m_{\omega}^{2}} \rho, \quad b_{03}=\frac{g_{\rho}}{2 m_{\rho}^{2}} \rho_{3} .
$$

The $\sigma$ meson field is determined through

$$
\frac{\partial P}{\partial \sigma}=\left(\frac{\partial P}{\partial M_{N}^{*}}\right)_{\mu_{i}, T} \frac{\partial M_{N}^{*}}{\partial \sigma}+\left(\frac{\partial P}{\partial \sigma}\right)_{M_{N}^{*}}=0 .
$$

We next calculate the binodal sections within this model.

\section{B. Binodal section}

Nuclear matter is not stable at all temperatures, proton fractions, and densities. If the Gibbs energy of a twocomponent phase is lower than the Gibbs energy of a onecomponent phase, the system will separate into two phases $[6,7]$. The stability criteria may be expressed by the following relations: 
TABLE I. Parameters used in the calculation for $R_{0}=0.6 \mathrm{fm}$. All masses and bag pressure are in $\mathrm{MeV}$.

\begin{tabular}{ccccccccc}
\hline \hline$m_{q}$ & $B^{1 / 4}$ & $Z$ & $m_{\sigma}$ & $m_{\omega}$ & $m_{\rho}$ & $g_{\omega}$ & $g_{\sigma}^{q}$ & $g_{\rho}$ \\
\hline 0 & 211.3 & 3.987 & 550 & 783 & 763 & 8.9539 & 5.981 & 8.655 \\
\hline \hline
\end{tabular}

$$
\begin{gathered}
C_{v}=\left(\frac{\partial u}{\partial T}\right)_{\rho, y_{p}}>0, \quad K=9\left(\frac{\partial P}{\partial \rho}\right)_{T, y_{p}}>0, \\
\left(\frac{\partial \mu_{p}}{\partial y_{p}}\right)_{T, P} \geqslant 0, \quad \text { and }\left(\frac{\partial \mu_{n}}{\partial y_{p}}\right)_{T, P} \leqslant 0,
\end{gathered}
$$

where $u=E / B$ is the energy per particle. These conditions guarantee thermodynamical stability (positive specific heat $C_{v}$ ), mechanical stability (positive compression modulus $K$ ), and diffusive stability (in a stable system, energy is required to change the proton concentration while the pressure and the temperature are kept constant). If any of these three criteria is violated, there will occur a phase separation.

The surface of two-phase coexistence, the binodal surface, is determined imposing the Gibbs conditions, namely, for a given temperature, the pressure and chemical potentials are equal for both phases. A discussion of the properties of phase separation in different models in terms of temperature, pressure, and proton fraction is most conveniently done by comparing the respective binodal surfaces. Understanding the properties of the mixed phase of nuclear matter is important for different systems both in astrophysics and nuclear physics. One example is the crust composition of a neutron star, which, near the surface, is thought to form a Coulomb lattice of nuclei immersed in an electron gas and to be responsible for the glitch phenomenon [5,24]. Also, in nuclear physics, highly excited nuclei created in heavy-ion collisions gives rise to multifragmentation, which is interpreted as a liquid-gas phase transition [3].

In order to obtain the proton and neutron chemical potentials in the two coexisting phases for a fixed pressure, we have used the geometrical construction $[6,7]$ with the neutron and proton chemical potential isobars as a function of proton fraction. This takes into account the diffusive stability conditions [Eq. (28)] and the Gibbs conditions for phase separation. For a given temperature, the binodal section, which contains points under the same pressure for different proton fractions, is obtained from the above conditions, simultaneously with the following equations:

$$
\begin{gathered}
P\left(\nu_{p}, \nu_{n}, M^{*}\right)=P\left(\nu_{p}^{\prime}, \nu_{n}^{\prime}, M^{* \prime}\right), \\
\mu_{i}\left(\nu_{p}, \nu_{n}, M^{*}\right)=\mu_{i}\left(\nu_{p}^{\prime}, \nu_{n}^{\prime}, M^{* \prime}\right), \quad i=p, n, \\
\left.\frac{\partial P}{\partial \sigma}\right|_{\sigma}=0, \quad \text { and }\left.\quad \frac{\partial P}{\partial \sigma}\right|_{\sigma^{\prime}}=0 .
\end{gathered}
$$

At this point, it is worth mentioning that the construction of the binodal section for the QMC is slightly more complicated than for the nonlinear Walecka model. This is because Eq. (15) has to be enforced in the numerical calculation for every $\sigma$.
The binodal section is formed by two branches, one corresponding to a gas phase and small proton fraction, and the other to a liquid phase and large proton fraction. It is, therefore, energetically favorable for nuclear matter to separate into a liquid phase with a large proton fraction (less asymmetric) and a gas phase with a small proton fraction (more asymmetric). This behavior is common to other relativistic mean field models and reflects the fact that the contribution from the $\rho$ meson gives a term in the energy per particle of the form $e_{\text {sym }}\left(\rho_{B}\right) \delta^{2}$ where the asymmetry parameter is $\delta$ $=\left(\rho_{n}-\rho_{p}\right) / \rho_{B}$ and the coefficient $e_{\text {sym }}$ increases with increasing $\rho_{B}$.

In the following section we will display the binodal section results and compare the same with different nonlinear relativistic models.

\section{RESULTS}

We start by fixing the free-space bag properties. We have used zero quark masses only and $R_{0}=0.6 \mathrm{fm}$ for the bag radius. There are two unknowns, $Z$ and the bag constant $B$. These are obtained as usual by fitting the nucleon mass $M$ $=939 \mathrm{MeV}$ and enforcing the stability condition for the bag. The values obtained for $Z$ and $B$ are displayed in Table I. The quark-meson coupling constants $g_{\sigma}^{q}, g_{\omega}=3 g_{\omega}^{q}$, and $g_{\rho}=g_{\rho}^{q}$ are fitted to obtain the correct saturation properties of nuclear matter, $E_{B} \equiv \epsilon / \rho-M=-15.7 \mathrm{MeV}$ at $\rho=\rho_{0}=0.15 \mathrm{fm}^{-3}$ and $a_{\text {sym }}=32.5 \mathrm{MeV}$. We take the standard values for the meson masses, also shown in Table I.

In the sequel we will frequently compare QMC to two different parametrizations of the NLWM, namely, the NL3 parametrization with nonlinear contributions from the scalar meson only [21] and the TM1 parametrization which includes quartic terms on scalar and vector mesons [22].

We first plot the energy per baryon as a function of the baryon density in Fig. 1 for symmetric nuclear matter, $y_{p}$ $=0.5$, for temperatures $0,4,8,12,16$, and $20 \mathrm{MeV}$. As expected, this functional at zero temperature has a minimum at the nuclear matter saturation density $\rho_{0}$, corresponding to a binding energy of $-15.7 \mathrm{MeV}$. As the temperature increases, the minimum shifts towards higher densities. This may be understood from the fact that to compensate for the larger kinetic energy, a larger value of $\rho$ is needed to give the minimum. For larger densities the nuclear repulsion effects take over, and the energy increases again. For higher temperatures, the minimum of the curves become positive, as in the usual NLWM. In Fig. 2, we plot the binding energy for neutron matter for temperatures 20, 50, and $100 \mathrm{MeV}$ and compare the results with TM1 and NL3 parametrizations of the NLWM. At higher temperatures, the QMC model gives a larger binding energy as compared to the Walecka models. The reason for this is the contribution for the thermal energy of the nucleon bag, which is absent in the Walecka model. 


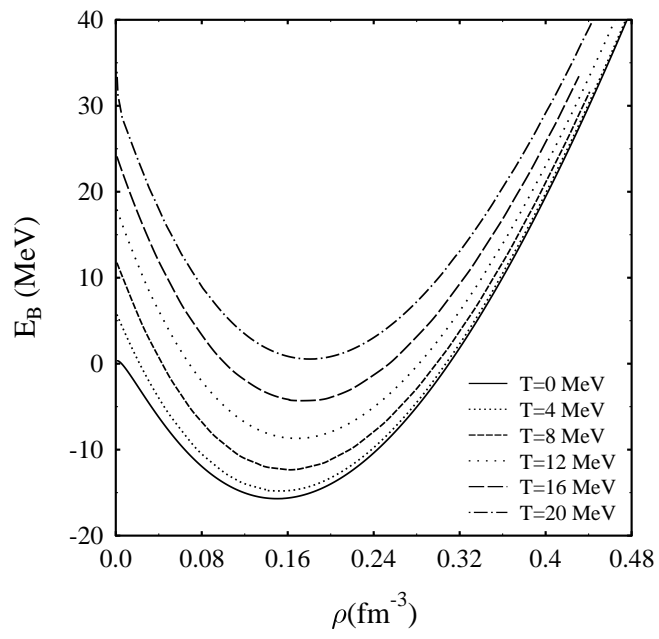

FIG. 1. Binding energy per nucleon $\left(E_{B}\right)$ as a function of nuclear matter density $\rho$ at temperatures $T=0,4,8,12,16$, and 20 $\mathrm{MeV}$.

This can be clearly seen from Fig. 3, where the baryon effective masses are plotted as a function of density for different temperatures. In the same figure, we have also shown the results of the parameter set NL3 and TM1 for comparison. The value of $M^{*}$ increases with increasing temperature. We have checked that at zero density and temperature $\sim 40 \mathrm{MeV}$, the QMC effective mass increases from 939 $\mathrm{MeV}$, in contrast to the calculation of hot nuclear matter using the Walecka model. The reason for this is that the $\sigma$ field is not strong at higher temperatures. Also, there is a significant contribution to the effective mass, arising from the thermal excitation of the quarks inside the nucleon bag, which adds to the mass of the nucleon at higher temperatures. This contribution, which is absent in the Walecka model calculation, appears dominant over the contribution from the $\sigma$ field, and the net effect is a rise in the effective mass. The behavior of the effective mass at high temperature obtained here is contrary to the results presented in Ref. [15],

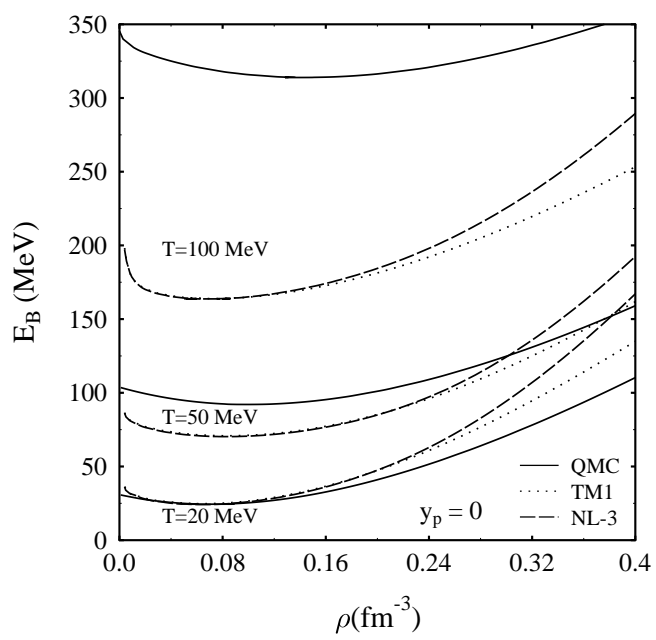

FIG. 2. Binding energy per nucleon $\left(E_{B}\right)$ as a function of nuclear matter density $\rho$ for neutron matter at temperatures $T$ $=20,50$, and $100 \mathrm{MeV}$, respectively.

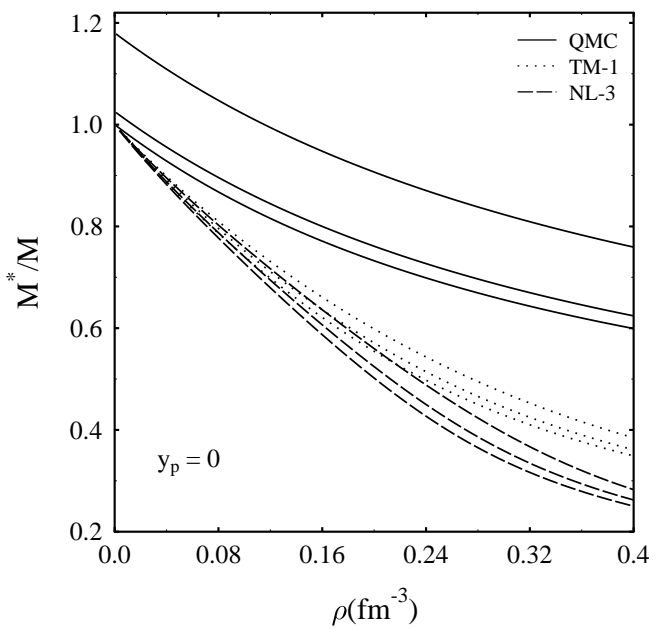

FIG. 3. Effective mass of the nucleon versus density at temperatures 20, 50, and $100 \mathrm{MeV}$ in QMC, NL3, and TM1 parameters.

where temperature was introduced only at the hadron level, and therefore the behavior of the effective mass with temperature, not surprisingly, is equivalent to the results of the Walecka-type of models.

In Fig. 4 we plot the effective radius in units of the free nucleon radius $R_{0}=0.6 \mathrm{fm}$ as a function of the baryon density for temperatures 20,50 , and $100 \mathrm{MeV}$. It is observed that the nucleon bag shrinks with the increase of temperature. A similar behavior is also predicted with the MQMC model [14]. Such an effect is also encountered using the thermal skyrmion [25] where a baryon shrinks with increase in temperature. We have also calculated the effective mass of the nucleon as a function of density at different temperatures for the QMC and the MQMC models. As already discussed in Refs. [17,18], the effective mass in the MQMC decreases faster with increase in $\rho$ than in the QMC model. However, also in the MQMC there is an increase of the effective mass with increase in temperature. A comparison between the QMC model and different versions of the modified MQMC has been performed in Ref. [18]. One important conclusion

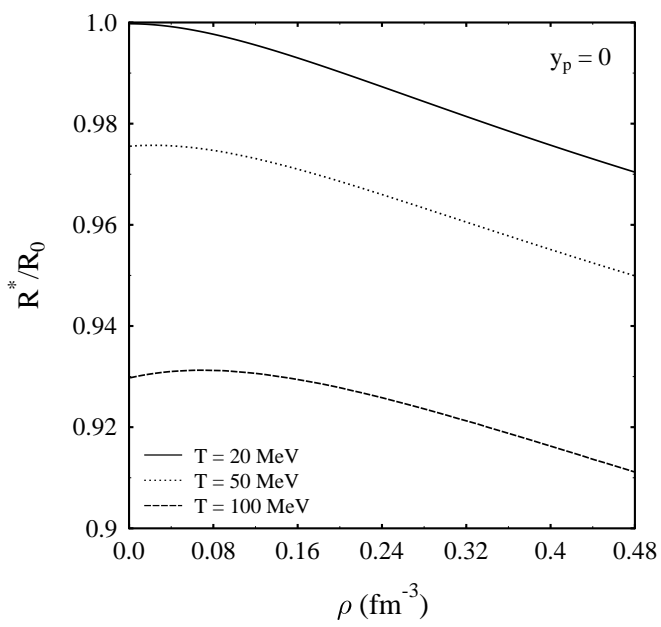

FIG. 4. The effective bag radius as a function of nuclear matter density $\rho$ for temperatures $T=20,50$, and $100 \mathrm{MeV}$, respectively. 


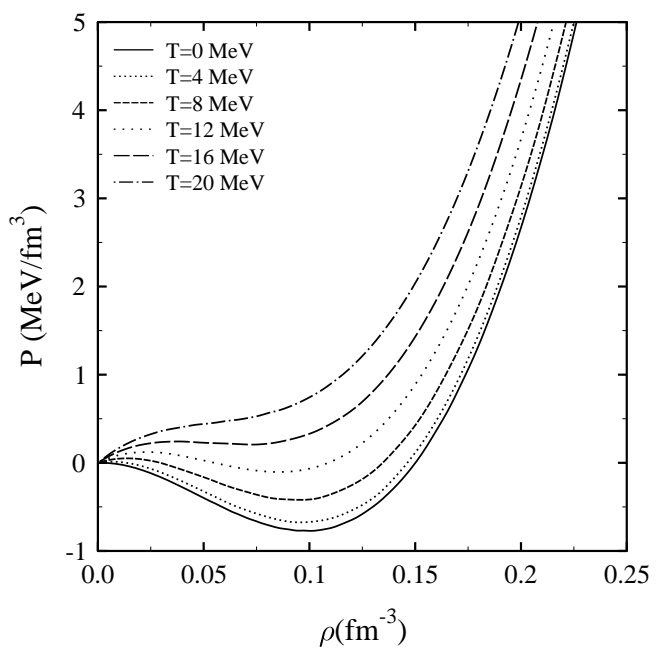

FIG. 5. Pressure $P$ as a function of nuclear matter density $\rho$ for symmetric matter at temperatures $T=0,4,8,12,16$, and $20 \mathrm{MeV}$, respectively.

was that, contrary to the QMC result, the bag radius increases with increase in density for all MQMC models. For densities not much larger than nuclear matter saturation density $\rho_{0}$, the bags start to overlap, which implies a breakdown of the model. On the other hand, the MQMC models contain sufficient free parameters, which allow a good reproduction of the ground state properties of nuclei. In the present work we were interested in describing propeties of nuclear matter for densities which go beyond the saturation density, and therefore have chosen to consider the QMC model.

The possible existence of a liquid-gas phase transition is determined by the pressure. We plot this quantity as a function of the baryon density $\rho$ for low temperatures in Fig. 5 for symmetric nuclear matter. At zero temperature, the pressure decreases with increase in density, reaches a minimum, and then increases and passes through $P=0$ at $\rho=\rho_{0}$, where

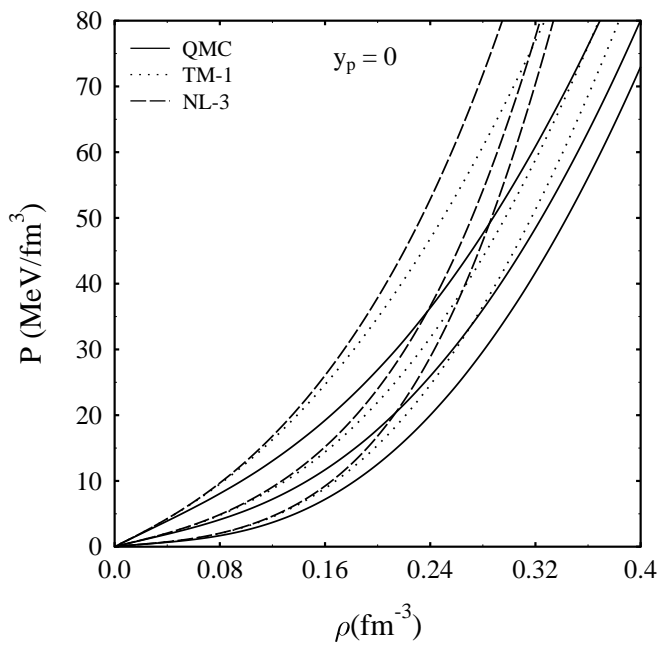

FIG. 6. Pressure $P$ as a function of nuclear matter density $\rho$ for neutron matter at temperatures $T=20,50$, and $100 \mathrm{MeV}$, respectively, from bottom to top. Note that the QMC model gives softer equation of state.

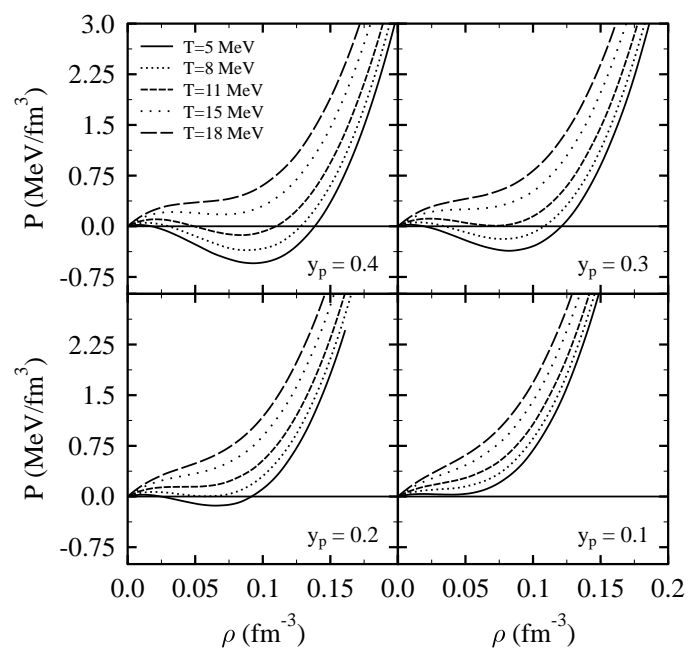

FIG. 7. Pressure $P$ as a function of nuclear matter density $\rho$ at different temperatures and different proton fractions.

the binding energy per nucleon is a minimum. A decrease of the pressure with increase in density corresponds to a negative compressibility, $K=9(\partial P / \partial \rho)$, and is a sign of mechanical instability. As the temperature increases, the region of mechanical instability decreases. At $T=17.7 \mathrm{MeV}$, the pocket disappears. This corresponds to the critical temperature defined by $(\partial P / \partial \rho)_{T, y_{p}}=0=\left(\partial^{2} P / \partial \rho^{2}\right)_{T, y_{p}}=0$, above which the liquid-gas phase transition is continuous. It is comparable to the values for the critical temperature obtained with Skyrme interactions [26] or relativistic models [7].

In Fig. 6, we plot the pressure as function of density for temperatures 20, 50, and $100 \mathrm{MeV}$. In the same figure we have also shown the results obtained with the NLWM of TM1 and NL3 parameter sets to compare. It is found that the QMC model gives a softer EOS, which is true also for any proton fraction. Although QMC gives a higher energy per particle than NL3 and TM1, the pressure, which corresponds to the derivative of the energy per particle with respect to density, does not increase so fast with increasing $\rho$.

In Fig. 7, the pressure is given as a function of the baryon density $\rho$ for different temperatures and different proton fractions. The region of mechanical instability decreases both with the increase of temperature and with the decrease of the proton fraction. This is clearly seen in Fig. 8, where the

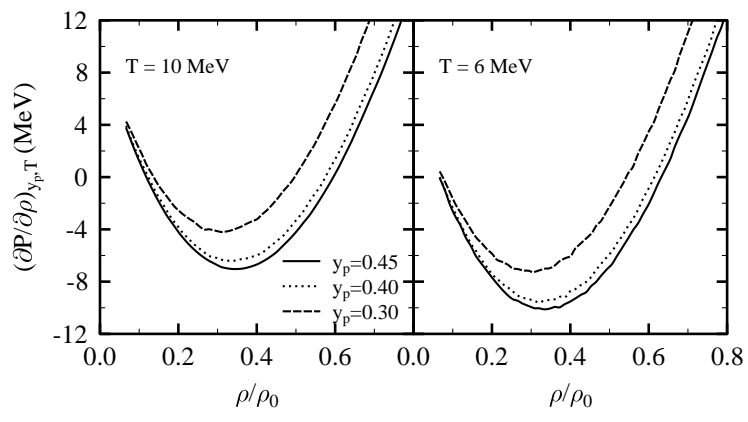

FIG. 8. $\partial P / \partial \rho$ as a function of the proton fraction. 


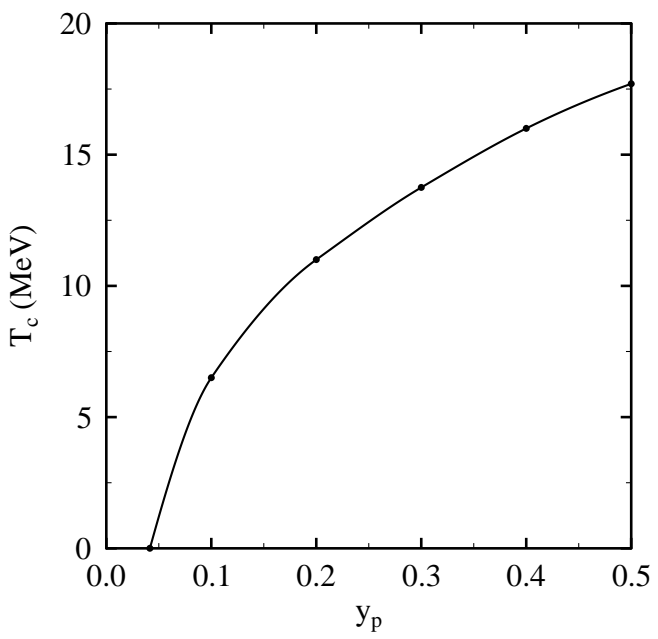

FIG. 9. Critical temperature as a function of the proton fraction.

quantity $(\partial P / \partial \rho)_{T, y_{p}}$ is shown for two different temperatures and three different proton fractions. The curve corresponding to $T=10 \mathrm{MeV}$ and $y_{p}=0.3$ has the smallest region with a negative value of the derivative $(\partial P / \partial \rho)_{T, y_{p}}$.

The behavior of the critical temperature with the proton fraction is shown in Fig. 9. It decreases rapidly with $y_{p}$, and goes to zero for $y_{p}=0.041$. Although there will be no mechanical instabilities above $T_{c}$, chemical instabilities can still develop above $T_{c}$. The present results are comparable to the ones discussed in Refs. [7,27,28].

The entropy per baryon is an important quantity to describe a collapsing massive star. During the whole process of the collapse, the entropy per baryon, including the contribution of baryons and leptons, remains low: it is of the order of $S / \rho \sim 1$ initially, increases slightly before neutrino trapping, and remains constant $(S / \rho \sim 1-2)$, afterwards [29]. According to different models, the contribution from the baryons alone changes from $80-90 \%$ at the saturation density to $50-$ $65 \%$ at ten times that density [1]. In Fig. 10 the entropy per

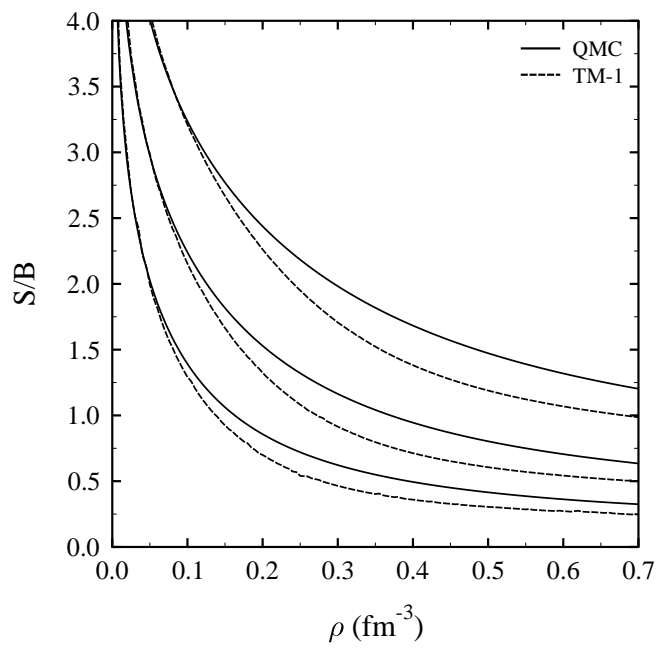

FIG. 10. Entropy per baryon, $S / B$, calculated within QMC and TM1, as a function of nuclear matter density $\rho$ for symmetric matter at temperatures $T=10,20$, and $40 \mathrm{MeV}$ from bottom to top.

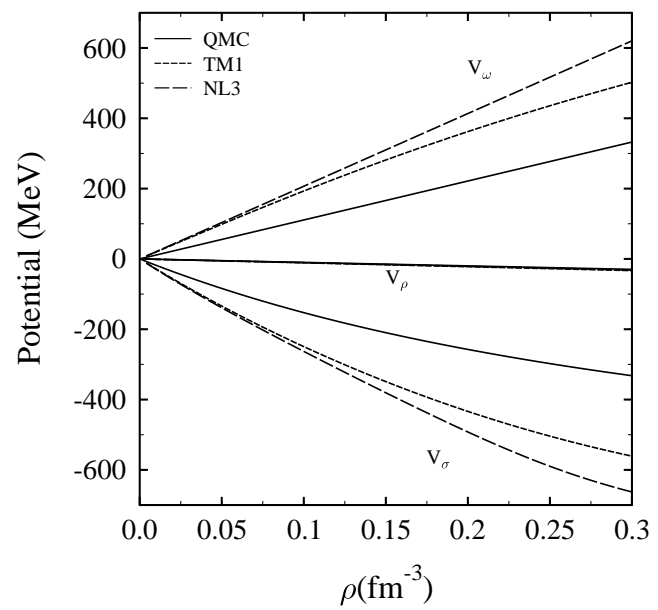

FIG. 11. Potentials versus nuclear matter density $\rho$ at temperature $=8 \mathrm{MeV}$ and $y_{p}=0.4$ in QMC, NL3, and TM1 parameters.

baryon is plotted and compared with the corresponding quantity calculated within the TM1 parametrization of NLWM. We conclude that QMC gives values compatible with the ones discussed in Ref. [1]. The entropy per baryon in QMC decreases slower than in TM1.

In Fig. 11, we plot the scalar and vector potentials as a function of the density for $T=8 \mathrm{MeV}$ and $y_{p}=0.4$. In the same figure, we have also shown the nonlinear Walecka model results. The $\omega$ and $\sigma$ contributions in the QMC are both weaker than the corresponding contributions in the NL3 and TM1 parametrizations of the NLWM. As a consequence, the QMC effective mass changes less with density than the TM1 and NL3 effective masses. This can also be confirmed in Fig. 3. A second consequence is a softer EOS due to the weaker $\omega$ field.

Isobars of nuclear pressure are plotted on the $\mu_{p}$ and $\mu_{n}$ space in Fig. 12 for $T=6 \mathrm{MeV}$ for two different pressures, $P=0.1$ and $0.063 \mathrm{MeV} / \mathrm{fm}^{3}$. The diffusively unstable regions can be seen clearly in chemical potential isobars in this figure. According to inequality (28), the region of negative

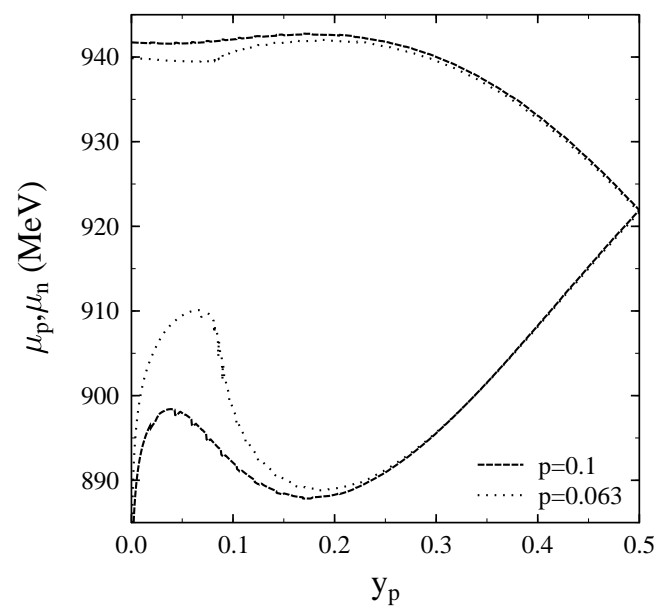

FIG. 12. Nuclear pressure isobars for the proton (lower) and neutron (upper) chemical potentials as a function of proton fraction for $P=0.1$ and $0.063 \mathrm{MeV} / \mathrm{fm}^{3}$. 


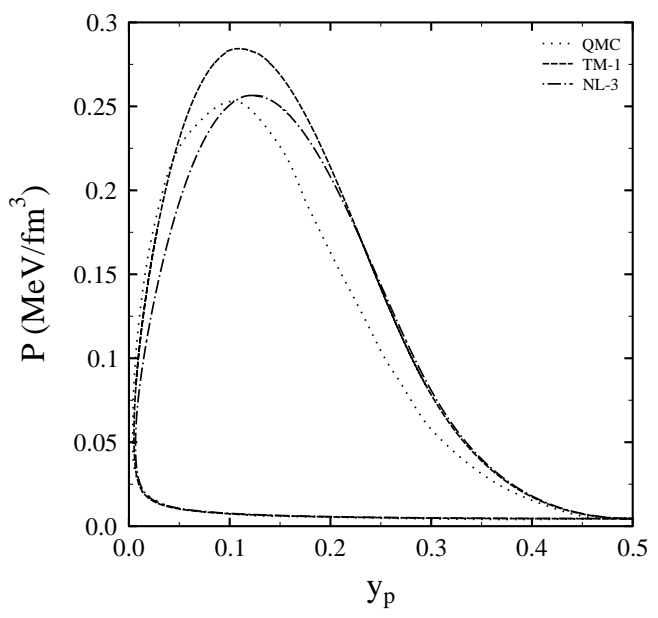

FIG. 13. The binodal section at $T=6 \mathrm{MeV}$ for QMC, NL3, and TM1.

(positive) slope for $\mu_{p}\left(\mu_{n}\right)$ is unstable. Violation of the stability criteria is an indication of phase separation. The surface of the two-phase coexistence in the $\left(p, T, y_{p}\right)$ space, the binodal surface, is obtained from conditions (29)-(31). In Fig. 13, we plot the binodal sections for the QMC and the NL3 and TM1 parametrizations, at $T=6 \mathrm{MeV}$. The critical point occurs for similar values of the pressure and proton fraction in all three models. For the TM1 parametrization, the critical pressure is $10 \%$ larger than the QMC critical pressure. On the other hand, the proton fraction for NL3 is $\sim 10 \%$ larger than the corresponding values for QMC. The largest difference between the models is in the shape: the region of configuration space where the phase separation is favorable is smaller in the QMC and occurs at smaller values of $y_{p}$ in the liquid phase. As a consequence, chemical instability occurs for smaller isospin asymmetries, which may have consequences in isospin fractionation [28]. The finite temperature results agree with the ones obtained within QMC at zero temperature [16]. For larger temperatures, the binodal has a similar shape but extends to smaller isospin asymmetries $[7,8]$.

\section{SUMMARY}

We now summarize the results and conclusions of the present work. We have studied asymmetric nuclear matter at finite temperatures using the QMC model that incorporates explicitly quark degrees of freedom. The results for the EOS were compared with the two nonlinear Walecka models, namely, NL3 and TM1 parametrizations. The mean effective fields $\sigma, \omega$, and $\rho$ are determined from the minimization of the thermodynamical potential, and the temperature dependent effective bag radius was calculated from the minimization of the effective mass of the nucleon mass of the bag. The thermal contributions of the quarks, which are absent in the Walecka model, are dominant and lead to a rise of the effective nucleon mass at finite temperatures. This is contrary to the results presented in Ref. [15], where temperature was introduced only at the hadron level, and therefore the behavior of the effective mass with temperature is equivalent to the results of Walecka-type models [11]. In the present calculation, the effective radius of the nucleon bag shrinks with the increase of temperature.

The equation of state, as derived here, is softer than the ones obtained within the nonlinear Walecka model for the NL3 and TM1 parametrizations, for all temperatures. The region of mechanical instability decreases with the increase of $T$ and decrease of $y_{p}$. Also, the critical temperature decreases with the proton fraction, from a maximum value of $T=17.7 \mathrm{MeV}$ at $y_{p}=0.5$ to $T=0 \mathrm{MeV}$ at $y_{p}=0.041$. We have shown that the potentials in the QMC are weaker than the corresponding ones in the NLWM for the NL3 and TM1 parametrizations, which imply a softer EOS and a weaker change of the effective mass with $\rho$.

We have also constructed and studied the binodal sections, which is essential for studying the liquid-gas phase transition and to understand under which conditions chemical instabilities develop, leading to isospin fractionation. It is clear from the binodal that the system prefers to separate into regions of higher density and smaller isospin asymmetry and regions of lower density and large isospin asymmetry $[7,8,28]$. The phase separation in the QMC occurs at smaller isospin asymmetry, corresponding to lower values of $y_{p}$, than the ones predicted by the NL3 and TM1 parametrizations of the NLWM. Extensions of the formalism presented here to droplet formation are currently under process.

\section{ACKNOWLEDGMENTS}

This work was partially supported by the CAPES, the CNPq, and the FAPESP (Brazil), and the FEDER, the GRICES, and the CFT (Portugal) under Contract No. POCTI/35308/FIS/2000. The facilities offered by the Center for Computational Physics, University of Coimbra, are warmly acknowledged.
[1] M. Prakash, I. Bombaci, M. Prakash, P.J. Ellis, J.M. Lattimer, and R. Knorren, Phys. Rep. 280, 1 (1997).

[2] M.C. Abreu et al., Phys. Lett. B 477, 28 (2000).

[3] J.A. Hauger et al., Phys. Rev. Lett. 77, 235 (1996); J. Phochodzalla et al., ibid. 75, 1040 (1995).

[4] C.J. Pethick, D.G. Ravenhall, and C.P. Lorenz, Nucl. Phys. A584, 675 (1995).
[5] C.P. Lorenz, D.G. Ravenhall, and C.J. Pethick, Phys. Rev. Lett. 70, 379 (1993).

[6] M. Barranco and J.R. Buchler, Phys. Rev. C 22, 1729 (1980).

[7] H. Müller and B.D. Serot, Phys. Rev. C 52, 2072 (1995).

[8] D.P. Menezes and C. Providencia, Nucl. Phys. A650, 283 (1999); Phys. Rev. C 60, 024313 (1999).

[9] P.A.M. Guichon, Phys. Lett. B 200, 235 (1988). 
[10] K. Saito and A.W. Thomas, Phys. Lett. B 327, 9 (1994); 335, 17 (1994); 363, 157 (1995); Phys. Rev. C 52, 2789 (1995); P.A.M. Guichon, K. Saito, E. Rodionov, and A.W. Thomas, Nucl. Phys. A601, 349 (1996); K. Saito, K. Tsushima, and A.W. Thomas, ibid. A609, 339 (1996); Phys. Rev. C 55, 2637 (1997); Phys. Lett. B 406, 287 (1997).

[11] B.D. Serot and J.D. Walecka, Int. J. Mod. Phys. E 8, 515 (1997); J.D. Walecka, Ann. Phys. (N.Y.) 83, 491 (1974).

[12] G. Krein, A.W. Thomas, and K. Tsushima, Nucl. Phys. A650, 313 (1999); M.E. Bracco, G. Krein, and M. Nielsen, Phys. Lett. B 432, 258 (1998).

[13] P.G. Blunden and G.A. Miller, Phys. Rev. C 54, 359 (1996); N. Barnea and T.S. Walhout, Nucl. Phys. A677, 367 (2000); H. Shen and H. Toki, Phys. Rev. C 61, 045205 (2000); P.K. Panda, R. Sahu, and C. Das, ibid. 60, 38801 (1999); P.K. Panda, M.E. Bracco, M. Chiapparini, E. Conte, and G. Krein, ibid. 65, 065206 (2002); P.K. Panda and F.L. Braghin, ibid. 66, 055207 (2002).

[14] P.K. Panda, A. Mishra, J.M. Eisenberg, and W. Greiner, Phys. Rev. C 56, 3134 (1997); I. Zakout and H.R. Jaqaman, ibid. 59, 962 (1999).

[15] H.Q. Song and R.K. Su, Phys. Lett. B 358, 179 (1995).

[16] G. Krein, D.P. Menezes, M. Nielsen, and C. Providencia, Nucl. Phys. A674, 125 (2000).

[17] X. Jin and B.K. Jennings, Phys. Lett. B 374, 13 (1996); Phys. Rev. C 54, 1427 (1996).

[18] H. Mueller and B.K. Jennings, Nucl. Phys. A640, 55 (1998); A626, 966 (1997).
[19] R.J. Furnstahl and B.D. Serot, Comments Nucl. Part. Phys. 2, A23 (2000); R.J. Furnstahl, B.D. Serot, and H.-B. Tang, Nucl. Phys. A598, 539 (1996); A614, 441 (1997); A618, 446 (1997).

[20] C.H. Johnson, D.J. Horen, and C. Mahaux, Phys. Rev. C 36, 2252 (1987).

[21] G.A. Lalazissis, J. König, and P. Ring, Phys. Rev. C 55, 540 (1997).

[22] K. Sumiyoshi, H. Kuwabara, and H. Toki, Nucl. Phys. A581, 725 (1995).

[23] K. Saito and A.W. Thomas, Phys. Lett. B 335, 17 (1994); T. Hatsuda, E.M. Henley, Th. Meissner, and G. Krein, Phys. Rev. C 49, 452 (1994); E.M. Henley and G. Krein, Phys. Rev. Lett. 62, 2586 (1989).

[24] N. K. Glendenning, Compact Stars (Springer-Verlag, New York, 2000).

[25] J. Dey and J.M. Eisenberg, Phys. Lett. B 334, 290 (1994); M.A. Nowak and I. Zahed, ibid. 230, 108 (1989); K.J. Eskola and K. Kajantie, Z. Phys. C 44, 347 (1989).

[26] H.R. Jaqaman, A.Z. Mekjian, and L. Zamick, Phys. Rev. C 29, 2067 (1984).

[27] S. Ray, J. Shamanna, and T.T.S. Kuo, Phys. Lett. B 392, 7 (1997).

[28] Bao-An Li, C.M. Ko, and W. Bauer, Int. J. Mod. Phys. E 7, 147 (1998); B.-A. Li, A.T. Sustich, Matt Tilley, and B. Zhang, Nucl. Phys. A699, 493 (2002).

[29] S. L. Shapiro and S. A. Teukolsky, Black Holes, White Dwarfs and Neutron Stars, the Physics of Compact Objects (Wiley, New York, 1983). 